American Journal of Applied Sciences 8 (8): 826-832, 2011

ISSN 1546-9239

(C) 2011 Science Publications

\title{
Study of Performance Analysis in Wired and Wireless Network
}

\author{
${ }^{1}$ V.B. Kirubanand and ${ }^{2}$ S. Palaniammal \\ ${ }^{1}$ Department of MCA, Sri Krishna College of Engineering \\ and Technology, 641008, Coimbatore \\ ${ }^{2}$ Department of Science and Humanities, VLB Janakiammal \\ College of Engineering and Technology 641042, Coimbatore
}

\begin{abstract}
Problem statement: The main intent of this study is to derive a high performance clientserver network using hub, switch, Bluetooth, WI-Fi and Wimax using the steadfast algorithms. Approach: This study mainly focuses on $\mathrm{M} / \mathrm{M}(\mathrm{a}, \mathrm{b}) / 1$ markovian model with adaboost algorithm and user selection algorithms to find performance on wired and wireless technologies in terms of service rate, arrival rate, Expected waiting time and Busy period. When comparing the wireless technologies with wired technologies in term of inter-arrival and inter-service time it has been found that the wireless technologies are better. Adaboost algorithm in between client-server is to deliver the data packets to the destination without any loss and also to boost the data packets to the destination when there occurs congestion in network. The main intention of user selection algorithm is to select the appropriate user one who have highest priority in the queue. If more than one user has the highest priority then a user is selected at random from among them with uniform probability. It also have been found that, in wireless technologies the data transaction in between client-server using Wimax technology is very efficient in $\mathrm{M} / \mathrm{M}(\mathrm{a}, \mathrm{b}) / 1$ for implementation with adaboost and user selection algorithm in terms of arrival rate, service rate, Expected waiting time and Busy period implementation. Results: The values obtained from the bluetooth technology can use for calculating the performance of other wireless technologies. Conclusion/Recommendation: In order to provide security for the data packets which flows inside the network, we use RSA algorithm which gives elevated security measures.
\end{abstract}

Key words: Client server, switch bluetooth, wimax technology, bulk service, wireless technologies, performance analysis, bluetooth technology, expected waiting, selection algorithm, steadfast algorithms, existing topology

\section{INTRODUCTION}

Client-server systems are becoming increasingly common in the world today as users move to networks of distributed, interacting computers. This process of work demands new performance modeling as the interaction in client server systems are more complex than the types supported by classic queueing Petri net solvers such as the modules contains as follows:

- Distribution time

- Root of specification

- Expected waiting time

- Expected busy period

Existing method: In the existing topology client computers were connected to server computers by means of anyone of the technology, i.e., either by Hub or Switch or Bluetooth or Wi-fi or Wimax or. Because of this the clients have to wait in queue for a long time to get service and also which gives the way to get congestion in the network. Congestion makes the client request packet or the server response packet bushed. The clients can't immediately change to other network while using the previous which cost high and low benefit. Another main drawback in the existing topology is that the hackers can easily hack the information. The congestion gives the way for the hackers to hack the information from the network. In the existing topology the clients have to wait in queue for a long time to get service, because the request of the clients were taken into server one by one for response, which uses FCFS (First Come First Served) rule as its default. Another main drawback of the existing topology is that, even though if the client request come with highest priority to get serviced, it have wait in queue till its turn come.

Corresponding Author: V.B. Kirubanand, Department of MCA, Sri Krishna College of Engineering and Technology, 641008, Coimbatore 
Am. J. Applied Sci., 8 (8): 826-832, 2011

\section{MATERIALS AND METHODS}

The layered queuing network solver, the model supports forwarding technique to clients (Barati, 2008; Bause, 1993; Wahsheh and Alves, 2007; Yang et al., 2007). The early replies are used to reduce the response time by replying to a client before all of its work at a server is completed. Activities represent the smallest unit of modeling. Franks (2000) Finally the solver has been extended to handle model with both homogeneous and heterogeneous threads within a task.

HUB: A Hub is a networking device that allows one to connect multiple PCs to a single network. Hubs may be based on Ethernet, Fire wire, or USB connections.

A network hub or repeater hub is a device for connecting multiple twisted pair or fiber optic Ethernet devices together and thus making them act as a single network segment. Hubs work at the physical layer (layer 1) of the OSI model (Fig. 1-8).

Switch: A network switch is a small hardware device that joins multiple computers together within one local Area Network (LAN). Technically, network switches operate at layer two (Data Link Layer) of the OSI model.

Bluetooth technology: Bluetooth is an open wireless protocol for exchanging data over short distances from fixed and mobile devices, creating personal Area Networks (PANs). It was originally conceived as a wireless alternative to RS232 data cables. It can connect several devices, overcoming problems of synchronization. The implication is that Bluetooth does the same with communications protocols, uniting them into one universal standard. \150.150.1.2/students $\backslash$ mcal07mcal07mca37\Bluetooth

Bluetooth uses a radio technology called frequencyhopping spread spectrum, which chops up the data being sent and transmits chunks of it on up to 79 frequencies. In its basic mode, the modulation is Gaussian Frequency-Shift Keying (GFSK). It can achieve a gross data rate of $1 \mathrm{Mb} / \mathrm{s}$.

Bluetooth provides a way to connect and exchange information between devices such as mobile phones, telephones, laptops, personal computers, printers, Global Positioning System (GPS) receivers, digital cameras and video game consoles through a secure, globally unlicensed Industrial, Scientific and Medical (ISM) $2.4 \mathrm{GHz}$ short-range radio frequency bandwidth. The Bluetooth specifications are developed and licensed by the Bluetooth Special Interest Group (SIG) (Sokullu and Karatepe, 2005). The Bluetooth SIG consists of companies in the areas of telecommunication, computing, networking and consumer electronics.

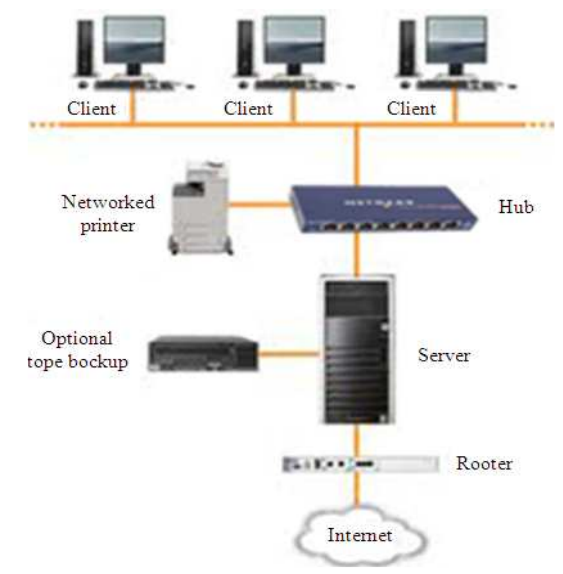

Fig. 1: The client and server connected in the transition devices with Hub



Fig. 2: The client and server connected in the transition devices with Switch

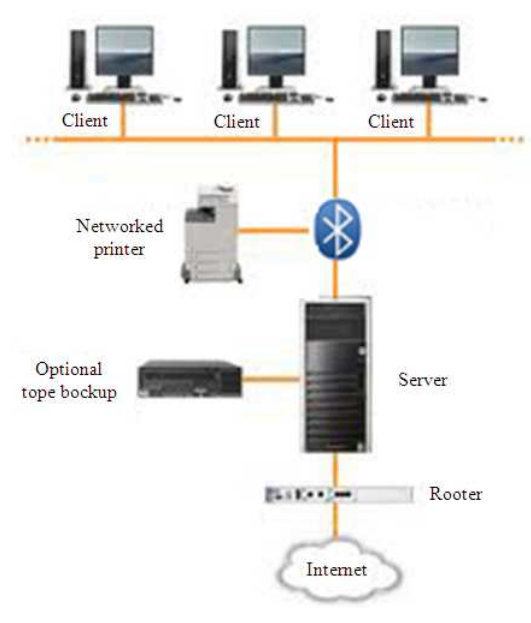

Fig. 3: The client and server connected in the transition devices with Bluetooth 


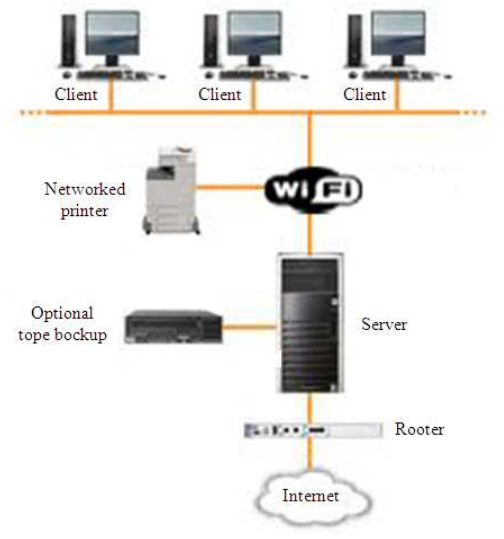

Fig. 4: The client and server connected in the transition devices with Wifi

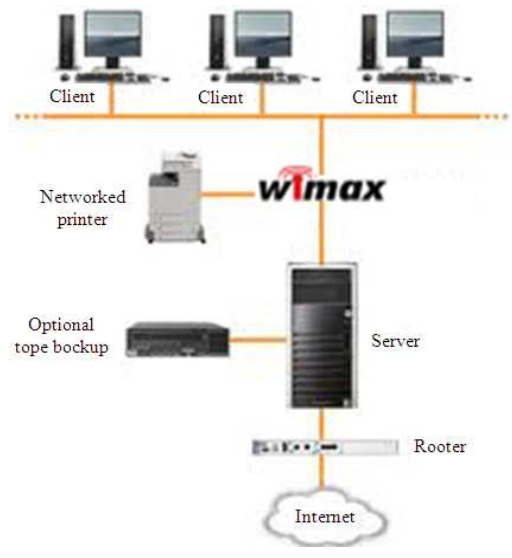

Fig. 5: The client and server connected in the transition devices with Wimax

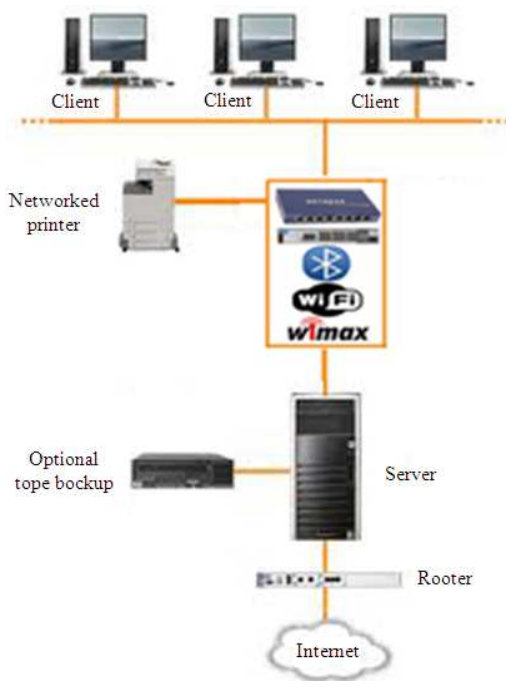

Fig. 6: The client and server connected in the hybrid model

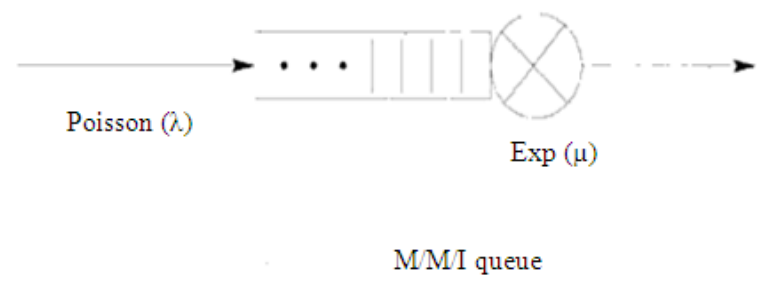

Fig. 7: Comparison of $M / M / 1$ andM/M(1,b)/1

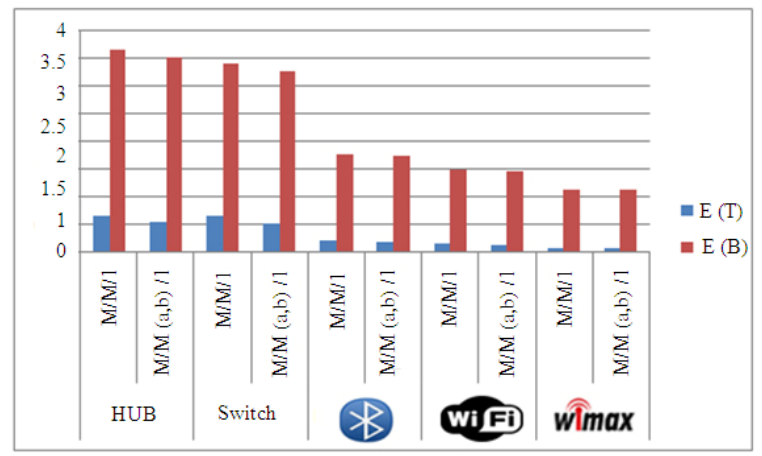

Fig. 8: E (T)-Expected waiting time: E (B)-Expected Busy Period

WI-fI technology: The name of a popular wireless networking technology that uses radio waves to provide wireless high-speed Internet and network connections. The Wi-Fi Alliance, the organization that owns the WiFi (registered trademark) term specifically defines WiFi as any 'Wireless Local Area Network (WLAN) products that are based on the Institute of Electrical and Electronics Engineers' (IEEE) 802.11 standards." Initially, Wi-Fi was used in place of only the $2.4 \mathrm{GHz}$ 802.11b standard, however the Wi-Fi Alliance has expanded the generic use of the Wi-Fi term to include any type of network or WLAN product based on any of the 802.11 standards, including $802.11 \mathrm{~b}, 802.11 \mathrm{a}$, dualband and so on, in an attempt to stop confusion about wireless LAN interoperability.

Wi-Fi works with no physical wired connection between sender and receiver by using Radio Frequency (RF) technology, a frequency within the electromagnetic spectrum associated with radio wave propagation. When an RF current is supplied to an antenna, an electromagnetic field is created that then is able to propagate through space. (New Avatars of Honeypot Attacks on WiFi Networks-Prabhash Dhyani) The cornerstone of any wireless network is an Access Point (AP). The primary job of an access point is to broadcast a wireless signal that computers can detect and "tune" into. In order to connect to an access point and join a wireless network, computers and devices must be equipped with wireless network adapters. 
Am. J. Applied Sci., 8 (8): 826-832, 2011

Table 1: Distribution time

\begin{tabular}{llllll}
\hline Technologies & Request/response & Ist system & 2nd system & 3nd system & Inter-arrival inter-service \\
\hline Hub & Req & 14.04 & 15.13 & 17.93 & $1 / 15.7$ \\
& Res & 1.5873 & 3.314 & 4.0009 & $1 / 2.9674$ \\
Switch & Req & 11.96 & 13.237 & 15.063 & $1 / 13.42$ \\
& Res & 1.0128 & 2.982 & 4.1571 & $1 / 2.7173$ \\
Bluetooth & Req & 11.71 & 12.931 & 14.869 & $1 / 13.17$ \\
& Res & 1.0039 & 1.809 & 1.9061 & $1 / 1.573$ \\
WIMi & Req & 11.067 & 12.682 & 14.531 & $1 / 12.76$ \\
& Res & 1.0017 & 1.2933 & 1.734 & $1 / 1.343$ \\
& Req & 10.997 & 12.485 & 14.459 & $1 / 12.647$ \\
\hline
\end{tabular}

Table 2: Comparison of wired and wireless nerwrk using M/M/1 and M/M (a,b)/1

\begin{tabular}{|c|c|c|c|c|c|c|c|c|}
\hline \multirow[b]{2}{*}{ Technologies } & \multirow{2}{*}{$\begin{array}{l}\text { Request } \\
(\lambda)\end{array}$} & \multirow{2}{*}{$\begin{array}{l}\text { Response } \\
(\mu)\end{array}$} & \multicolumn{3}{|l|}{ M/M/I } & \multicolumn{3}{|c|}{$\mathrm{M} / \mathrm{M}(\mathrm{a}, \mathrm{b}) / 1$} \\
\hline & & & $\mathrm{r}$ & $\mathrm{E}(\mathrm{T})$ & $\mathrm{B}(\mathrm{T})$ & $\mathrm{r}$ & $\mathrm{E}(\mathrm{T})$ & $\mathrm{B}(\mathrm{T})$ \\
\hline Hub & 0.06369 & 0.33699 & 0.18901 & 0.66341 & 3.65904 & 0.15949 & 0.54694 & 3.53053 \\
\hline Switch & 0.07452 & 0.36801 & 0.20249 & 0.65751 & 3.40725 & 0.16907 & 0.53501 & 3.27021 \\
\hline Bluetooth & 0.07593 & 0.63572 & 0.11944 & 0.21001 & 1.78638 & 0.10682 & 0.18578 & 1.76115 \\
\hline WI-FI & 0.07837 & 0.74460 & 0.10525 & 0.15607 & 1.50098 & 0.0953 & 0.14008 & 1.48447 \\
\hline Wimax & 0.07907 & 0.95877 & 0.08247 & 0.09306 & 1.13675 & 0.07622 & 0.08552 & 1.12906 \\
\hline
\end{tabular}

Wimax technology: WiMax can be used for wireless networking like the popular WiFi. WiMax, a secondgeneration protocol, allows higher data rates over longer distances, efficient use of bandwidth and avoids interference almost at minimum (Salama, 0000).

WiMax can also be used in the following applications:

Automatic teller machines, Online gaming, Multimedia communication, Medical applications, Vehicular data and voice, Sensor networks, Telematics and telemetry. Mobile transmission of information in emergency situations, Real-time monitoring, alerting and controlling the process of dangerous works. Wireless transmission of informaion of fingerprints, photos, Warrants and other images to and from law-enforcemen field personnel (Queuing Petri Nets.-F. Bause).

IEEE standards: WiMax is based on IEEE 802.16.

Range: WiMAX antenna is expected to have a range of up to 40 miles with speeds of $70 \mathrm{Mbps}$ or more.

Scalability: WiMax is designed to efficiently support from one to hundreds of Consumer Premises Equipments (CPE) s, with unlimited subscribers behind each CPE. Flexible channel sizes from $1.5 \mathrm{MHz}$ to $20 \mathrm{MHz}$.

Bit rate: $\mathrm{Wi}-\mathrm{Fi}$ works at $2.7 \mathrm{bps} / \mathrm{Hz}$ and can peak up to $54 \mathrm{Mbps}$ in $20 \mathrm{Mhz}$ channel.But other technologies like WiMax works at $5 \mathrm{bps} / \mathrm{Hz}$ and can peak up to $100 \mathrm{Mbps}$ in a $20 \mathrm{Mhz}$ channel
Quality of service: Other wireless technology does not guarantee any QoS but WiMax provide several level of QoS. Also WiMax can bring the underlying Internet Connection needed to service other local wireless networks (Singh and Sohi, 2008).

Proposal method: In the proposed system the user gets much benefit because of the steadfast algorithms. In the proposed system the integration both wired and wireless technology, i.e., Hub, Switch, Bluetooth, Wifi, Wimax in between client and server gives a new Hybrid model which provide the best performance when compared to existing topology. The main intent of hybrid model is to avoid the congestion in network and also to save the client waiting time in the queue. The data transaction between client-server in hybrid model is very efficient in time and cost. Because of the Markovian M/M (a, b)/1 bulk service rule, client gets much benefit when comparing with $M / M / 1$. While using $M / M(a, b) / 1$ bulk service rule, client request are taken to server for service in a bulk (more than one), not one by one. Because of this, clients waiting time in queue are abridged a lot and the clients don't need to wait a long in queue to get services( Fig.7-8).

Hybrid model network: The work station-server model is the most widely used model for building distributed computing system (Breuer and Baum, 2005).

This is because a large number of computer users only perform only simple interactive task such as sending packets through email, LAN, WAN, MAN. The study station-server model is idle for such simple 
usage to transaction the data in between client and the server (Freund and Schapire, 1999).

\section{RESULTS AND DISCUSSION}

Adaboost algorithm: Adaboost, short for Adaptive Boosting, is a branch of boosting algorithm which specifies the weight adaptively. (Jayakumar and Gopinath, 2008) The AdaBoost algorithm, introduced in by Freund and Schapire (1999), solved many of the practical difficulties of the earlier boosting algorithms.

The algorithm takes as input a training set $\left(\mathrm{x}_{1}, \mathrm{y}_{1}\right)$ ,$\ldots\left(\left(\mathrm{x}_{\mathrm{m}}, \mathrm{y}_{\mathrm{m}}\right)\right)$ where each $\mathrm{x}_{\mathrm{i}}$ is an object in the input training data belonging to the class $y_{i}$ and each $y_{i}$ is either +1 or -1 . Kounev and Buchmann (2006). In this study we dont extend AdaBoost to multi-class problems. Medhi (1994). The weak classifiers $C_{1}, \ldots C_{T}$ are also provided for AdaBoost. Each $\mathrm{C}_{\mathrm{tb}}$ is a function which inputs an object $x_{i}$ and outputs a guess $\bar{y}_{i}$. The guessed class $\bar{y}_{i}$ must be slightly correlated with the true class $y_{i}$. AdaBoost calls weak the classifier $C_{t}$ in a series of rounds $t=1, \ldots, T$ and calculates a weight $a_{t}$ for $\mathrm{C}_{\mathrm{t}}$ according to its classification error $\mathrm{e}_{\mathrm{t}}$.

The algorithm also keeps weights $\mathrm{w}_{1, \ldots} \mathrm{w}_{\mathrm{m}}$ for the training objects. Initially all weights are set equally, but on each round, the weights of incorrectly classified objects are increased so that the weak classifiers in the future rounds are forced to focus on hard examples in the training set.

Markovian algorithm (RSA): In this section we introduce the definition of the markov process associated with queueing networks. The detailed definition allows us to define an exact solution method to evaluate the joint queue length distribution of nodes in steady-state conditions and to evaluate average performance indices. External arrivals are assumed to be poisson whereas the service time distribution of each service center is defined using of phase-type distributions. We assume independence between successive services of the nodes and the interarrival times. Medhi (1994) Under these assumptions, the queueing network can be represented by a continuoustime homogeneous markov chain. The stationary and transient behavior of the network can be analyzed by this underlying markov process.

M/m/1 Markovian model: The M/M/1queue in continuous time is defined by the following characteristics: the arrival process is a Poisson process with some rate $\lambda>0$. The service times are iid and distributed exponentially with service rate $\mu>0$. There is one server and the service discipline in first come first served (Breuer and Baum, 2005).
M/M (a,b)/1 Bulk service rule: There could be a number of rules according to which batches for bulk service may be formed. The following are the types of bulk service rules:

- Dhyani (2009) considers that units are served in batches of not more than $b$. if immediately after the completion of a service, the server finds more than $\mathrm{b}$ units waiting, it takes a batch of $\mathrm{b}$ units for service while others wai If it finds $r$ units $\left(0 \leq \tau \leq_{b}\right)$. It takes all $\mathrm{r}$ units in a batch for service. Franks (2000) consider the same rule with the restriction that $r \neq 0\left(10 \leq \tau \leq{ }_{b}\right)$ i.e., the service facility stops until a unit arrives. This rule will be called the usual bulk service rule, while Bailey's rule will be its modified type (it will be also called bulk service rule with intermittently available server) Jaiswal points out that the distribution of the queue length for the modified rule can be obtained from that of the usual rule (Medhi, 1994)

- The rule with a Gated batch size $\mathrm{k}$ has been considered. Kounev et al. (2006). Ln this case the server waits until there are $\mathrm{k}$ units in a batch. if there are more than $\mathrm{k}$ waiting then the server becomes free, he takes a batch of $\mathrm{k}$ for service while others wait

Comparison of $\mathbf{m} / \mathbf{m} / \mathbf{1}$ and $\mathbf{m} / \mathbf{m}(a, b) / 1$ : In the existing topology it was mathematically proved that $\mathrm{M} / \mathrm{M} /(\mathrm{a}, \mathrm{b}) / 1$ model was better when compared to $\mathrm{M} / \mathrm{M} / 1$ model in queuing model. But in this proposed topology, it was proved in the computer theoretically (Jayakumar and Gopinath, 2008).

\section{Performance measures: \\ Root of specification: \\ For M/M/1:}

$$
r=\frac{\lambda}{\mu}
$$

For M/M (a,b)/1:

$$
r=\mu z^{b+1}-(\lambda+\mu) z+\lambda
$$

Probability that the server is idle:

$$
\mathrm{P} 0,0=\frac{(1-r)}{1-r+(\lambda / \mu)}=\frac{(1-r)^{2}}{(1-r)^{2}+r\left(1-r^{b}\right)}
$$

Probability that the server is busy and $\mathrm{n}$ units in the system: 


$$
p_{1, n} \frac{(1-r)\left(1-r^{b}\right)}{(1-r)^{2}+r\left(1-r^{b}\right)} r^{n+1}, n=0,1,2
$$

Waiting time density:

$$
v(t)=\frac{\lambda \mathrm{p} 0,0}{(1-\mathrm{r})}\left\{\left(1-\mathrm{r}^{\mathrm{b}}\right) \exp \left(\mu\left(1-\mathrm{r}^{\mathrm{b}}\right) \mathrm{t}\right\}\right.
$$

Expected waiting time density:

$$
E(T)=\frac{r}{\left.\mu\left(1-r^{b}\right)+r\left(1-r^{b}\right)\right]}
$$

Expected busy period:

$$
E(B)=\frac{1}{\mu(1-r)}
$$

From the above formulas we are calculating the Performance of Client-Server model.

A comparative study of the wireless network has been done with help of the Markova bulk service rule, adaboost with RSA algorithm, in the local area network with the bandwidth of $100 \mathrm{mbps}$.

Smallest weights, w (i) and w (j), are merged into a new tree whose weight is $w(i)+w(j)$ and whose root has two children which are the sub trees represented by $w(i)$ and $w(j)$. The weights $w(i)$ and $w(j)$ are removed from the list and w (i) +w (j) is inserted into the list.

\section{CONCLUSSION}

This process continues until the weight list contains a single value. If, at any time, there is more than one way to choose a smallest pair of weights, any such pair may be chosen. In the Huffman algorithm, the process should begin with a non Increasing list of weights because it provides a more efficient implementation.

For encryption and decryption the algorithm uses the below formula.

For encryption: Public key (but not your private key) from a central administrator and encrypt a message to the second user using your public key. When the second user receives it, he/she decrypt it with first user's private key. (Gurumurthy, 2010) In addition to encrypting messages (which ensures privacy), the second user can authenticate himself to the first user by using second user private key to encrypt a digital certificate. When the first users receive it, he can use second user's public key to decrypt it:
- Use the public key $\mathrm{E}$ and $\mathrm{n}$ for encrypting the message in order to form the cipher text (Stajano and Harris, 2008)

- The message M must be converted in the form of integer i.e., it may br converted into ASCII value

- Check whether the integer representation of message $M$ must satisfy the form $M<n$, otherwise its not possible to decrypt the original message

- The calculation of cipher text $\mathrm{C}$ from the integer representation of the message $\mathrm{M}$ is $\mathrm{C}=\mathrm{M}^{\mathrm{E}} \bmod (\mathrm{N})$

For decryption: Use the private key $\mathrm{E}$ and $\mathrm{n}$ for decrypting the cipher text $\mathrm{C}$ in order to get the original message $\mathrm{M}$ :

- The cipher text must be in the form of integer.

- The calculation of decryption process is $\mathrm{M}=\mathrm{C}^{\mathrm{D}}$ $\bmod (\mathrm{n})$

- The calculated value M needs to convert into corresponding character code

The private key is used to decrypt text that has been encrypted with the public key. Thus, if I send you a message, the first user can find out your

Results of this work: Thus this research work concludes that the performance of network sharing client-server model is better in $\mathrm{M} / \mathrm{M}(\mathrm{a}, \mathrm{b}) / 1$ model when compared to $\mathrm{M} / \mathrm{M} / 1$ model by applying Steadfast algorithms. Also it has been found that the Wimax is better implementation for better performance when compared with wired and wireless technologies and we hope to motivate further research in this area

\section{REFERENCES}

Barati, A., 2008. Decreasing overhead and power consuming in Ad-hoc networks by proposal a novel routing alogorithm. J. Comput. Sci., 4: 427-436. DOI: 10.3844 jessp.2008.427.436

Bause, F., 1993. Queuing petri nets- a formalism for the combined qualitative and quantitative analysis of systems. Proceedings., 5th International Workshop Petri Nets and Performance Models, Oct. 19-22, Toulouse, France, pp: 14-23. DOI: 10.1109/PNPM.1993.393439

Breuer, L. and D. Baum, 2005. An Introduction to Queueing Theory: And Matrix-Analytic Methods. 1st Edn., Springer, SBN-10: 9781402036309, pp: 271. 
Dhyani, P., 2009. New Avatars of Honeypot Attacks on $\mathrm{WiFi}$

Networks. http://www.security.iitk.ac.in/contents/events/work shops/iitkhack09/papers/prabhash.pdf

Franks, R.G., 2000. Performance analysis of distributed server systems. Carleton University Ottawa, Ont., Canada, Canada. http://portal.acm.org/citation.cfm?id=931221

Freund, Y. and R.E. Schapire, 1999. A Short Introduction to Boosting. J. Japan. Soci. Artifici. Intell., 14: 771-780. http://citeseerx.ist.psu.edu/viewdoc/download?doi= 10.1.1.93.5148\&rep=rep1\&type=pdf

Gurumurthy, S., 2010. Network security. Proceedings of the World Congress on Engineering, Jun. 30July 2, London, UK., pp: 460-464. http://www.iaeng.org/publication/WCE2010/WCE 2010_pp460-464.pdf

Jayakumar, G. and G. Gopinath, 2008. Performance comparison of two on demand routing protocols for Ad-hoc networks based on random way point mobility model. Am. J. Applied Sci., 5: 659-664. http://citeseerx.ist.psu.edu/viewdoc/download?doi= 10.1.1.105.980\&rep=rep1\&type $=$ pdf

Kounev, S. and A. Buchmann, 2006. Performance modeling of distributed e-business application using queueing petri nets. IEEE Trans. Software Eng., 32: 486-502. DOI: 10.1109/TSE.2006.69

Kounev, S., C. Dutz and A. Buchmann, 2006. Queueing petri net modeling environment. Proceedings of the 3rd International Conference on the Quantitative Evaluation of Systems, Sept. 11-14, Riverside, California, pp: 115-116. http://doi.ieeecomputersociety.org/10.1109/QEST.2 006.44
Medhi, J., 1994. Stochastic Processes. 2nd Edn., New Age International Publishers, ISBN: 8122405495, pp: 598.

Salama, A.S., 2008. Topologies indexed by relation with application. J. Comput. Sci., 4: 877-887.

Singh, G. and B.S. Sohi, 2008. Experimentsl Evaluation of medium access schemes in 802.11 wireless networks. J. Comput. Sci., 4: 888-896. DOI: 10.3844/jcssp.2008.888.896

Sokullu, R. and E. Karatepe, 2005. Performance evaluation of bluetooth links in the presence of specific types of interference. Proc. World Acad. Sci., Eng. Technol., 3: 17-21. http://citeseerx.ist.psu.edu/viewdoc/download?doi= 10.1.1.119.4600\&rep=rep1\&type $=$ pdf

Stajano, F. and W. Harris, 2008. Romantic Cryptrography.

http://www.anagram.com/jcrap/Volume_7/Romanc e.pdf

Wahsheh, L.A. and J. Alves, 2007. Policy based security for wireless components in high assurance computer system. J. Comput. Sci., 3: 726-735. http://citeseerx.ist.psu.edu/viewdoc/download?doi= 10.1.1.165.7851\&rep=rep1\&type $=$ pdf

Yang, Z., A. Mohammed, T. Huh and D. Grace, 2007. Optimizing downlink coexistence performance of WiMAX services in HAP and terrestrial deployments in shared frequency bands. Proceedings of the International Waveform Diversity and Design Conference, Jun. 4-8, Pisa, pp: 79-82. DOI: 10.1109/WDDC.2007.4339384 